\title{
Processed and Unprocessed Ices in Circumstellar Disks
}

\author{
Klaus Pontoppidan \& Ewine van Dishoeck \\ Leiden Observatory, P. O. Box 9513, 2300 RA Leiden, Netherlands
}

Emmanuel Dartois

IAS, Bât 121, Université Paris XI, 91405 Orsay Cedex, France

Wing-Fai Thi

Astronomical Institute "Anton Pannekoek", University of Amsterdam, Kruislaan 403, 1098 SJ Amsterdam, Netherlands

\begin{abstract}
We present 3-5 $\mu \mathrm{m}$ VLT-ISAAC spectroscopy showing the presence of methanol ices in edge-on disks of young embedded stars. Examples include the disks of L1489 IRS in Taurus and CRBR 2422.8-3423 in Ophiuchus, the last of which has the highest column density of solid $\mathrm{CO}$ known toward a YSO. Several additional low-mass sources in the Serpens and Chameleon molecular clouds exhibit abundant solid methanol although it is not clear if the ice is associated with a disk or with the envelope. These are the first detections of solid methanol in the disks and circumstellar environments of embedded young low-mass stars providing evidence that complex molecular species previously observed only in the solid state toward high-mass star forming regions are also present near solar-type young stars. The constraints on the formation mechanisms of methanol and the chemical evolution of ices as the material is incorporated into circumstellar disks are discussed.
\end{abstract}

\section{Introduction}

The presence of abundant ices in the interstellar medium has long been firmly established and its connection to the ices present in solar system comets has been the subject of some discussion. Most of the major constituents of interstellar grain mantles have now been firmly identified, mostly due to the ISO mission and extensive laboratory work. However, only recently have attempts been made to observe the ices in the context of their astrophysical environments. Models indicate that ices may undergo significant transformation when material from a protostellar envelope is incorporated into a disk, either through shock evaporation (Charnley and Rogers, 2003) and recondensation or through growth of ice mantles under different densities and temperatures than those of the quiescent interstellar medium (e.g., Aikawa et al 2003).

In this contribution, new direct observations of ices in circumstellar disks are described and compared to ices more likely to be associated with circumstellar envelopes and quiescent molecular cloud material. 


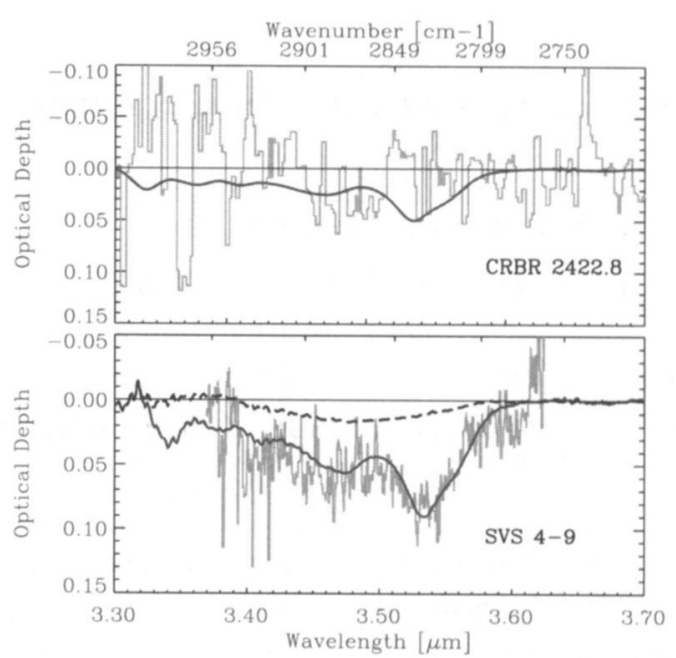

Figure 1. Top: Upper limit of the methanol $3.53 \mu \mathrm{m}$ band toward the edgeon disk CRBR $2422.8-3423\left(N\left(\mathrm{CH}_{3} \mathrm{OH}\right) / N\left(\mathrm{H}_{2} \mathrm{O}\right) \leq 0.06\right)$. Bottom: VLTISAAC detection of methanol toward a YSO (SVS 4-9) which probes dense envelope material and an outflow rather than a disk $\left(N\left(\mathrm{CH}_{3} \mathrm{OH}\right) / N\left(\mathrm{H}_{2} \mathrm{O}\right)=\right.$ $0.25)$. The dashed curves show a template for the $3.47 \mu \mathrm{m}$ feature. The solid curves show a sum of the $3.47 \mu \mathrm{m}$ template and a laboratory spectrum of an $\mathrm{H}_{2} \mathrm{O}: \mathrm{CH}_{3} \mathrm{OH}: \mathrm{H}_{2} \mathrm{CO}=1: 1: 1$ mixture at $10 \mathrm{~K}$.

\section{Observations of ices in disks}

Mid-infrared spectroscopy of nearly edge-on circumstellar disks around young stars probe a line of sight passing through the cold disk mid-planes where ices are expected to exist. As part of a 3-5 $\mu \mathrm{m}$ spectroscopic survey of more than 40 young low mass stars (van Dishoeck et al. 2003) a small selection of sources with edge-on disks have been included. Previously, the stretching mode of solid $\mathrm{CO}$ at $4.67 \mu \mathrm{m}$ have been analysed in this manner for the low mass edge-on disk sources L1489 IR (Boogert et al. 2002) and CRBR 2422.8-3423 (Thi et al. 2002).

Here we present the results from a search for solid methanol toward these two sources. Methanol $\left(\mathrm{CH}_{3} \mathrm{OH}\right)$ is believed to be formed primarily in the solid state through successive hydrogenation of $\mathrm{CO}$. The formation efficiency of this species is particularly interesting due to its significant role in the gasphase formation of more complex organic molecules. In Fig. 1 a VLT-ISAAC spectrum of the $3.53 \mu \mathrm{m}$ region of CRBR $2422.8-3423$ is presented showing a non-detection of solid methanol with an upper limit of $6 \%$ with respect to water ice. For comparison a strong methanol band observed toward the low mass YSO SVS 4-9 in the Serpens cloud core is shown below (Pontoppidan et al. 2003).

In Fig. 2 the same spectral region is shown of L1489. The band is clearly dominated by the " $3.47 \mu \mathrm{m}$ feature", which is unrelated to the methanol band. The presence of this band makes it difficult to determine the abundance of solid methanol even if the spectrum is of high quality. The spectrum of another low mass source, IRS 42, is also shown as an example of a good upper limit 


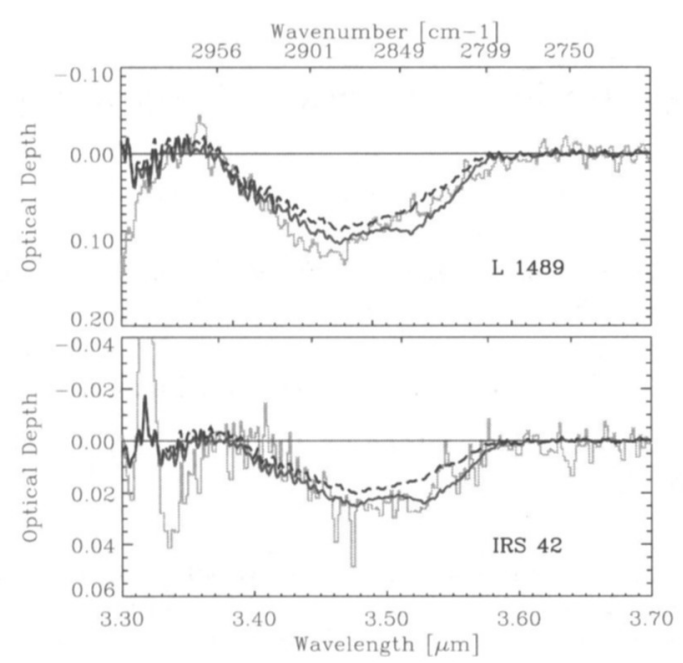

Figure 2. Same as Fig. 1. Top: The deep $3.47 \mu \mathrm{m}$ feature of the edge-on disk source L1489. The solid curve corresponds to a methanol content of $5 \%$. Bottom: A comparison with IRS 42 in the Ophiuchi cloud core thought to probe mostly quiescent molecular cloud material. The solid curve corresponds to a methanol content of $4 \%$.

of $\lesssim 4 \%$ methanol with respect to water. We find in general that about $10 \%$ of our observed lines of sight contain ice with $20 \%$ or more methanol, while only upper limits of $2-10 \%$ methanol are available for the rest. This implies that the methanol content of specific grain mantles may vary with an order of magnitude, depending on the environment. We have not found firm evidence of abundant methanol in the circumstellar disk material observed so far, but our upper limits are still consistent with cometary abundances. If methanol-rich ices from protostellar envelopes have been included in these disks, the methanol must have been diluted or destroyed during the process of disk-formation.

\section{References}

Aikawa, Y., Ohashi, N. \& Herbst, E. 2003, ApJ, 593, 906

Boogert, A. C. A., Hogerheijde, M. R. \& Blake, G. A. 2002 ApJ, 568, 761

Charnley, S.B. \& Rodgers, S.D. 2003, This volume, JD 14

van Dishoeck, E. F., Dartois, E., Pontoppidan, K. M. et al. 2003, ESO Messenger, 113, 49

Pontoppidan, K. M., Dartois, E., van Dishoeck, E. F., Thi, W.-F. \& d'Hendecourt, L. 2003, A\&A, 404, 17

Thi, W. F., Pontoppidan, K. M., van Dishoeck, E. F., Dartois, E. \& d'Hendecourt, L. 2002, A\&A, 394, 27 